\title{
A METHOD TO EVALUATE THE FIELD-SHAPE MULTIPOLES INDUCED BY COIL DEFORMATIONS
}

\begin{abstract}
P. Ferracin, W. Scandale ${ }^{*}$, E. Todesco, P. Tropea, Abstract

A semi-analytical method to evaluate the effect of coil deformations on the field-shape imperfections of the LHC dipole is presented. The deformation induced by the collaring procedure and by the thermal stresses is evaluated numerically with a finite element code. The vector field of mechanical displacements is approximated with truncated Taylor and Fourier series. The fitting function agrees with the numerical data to within less that $10 \mu \mathrm{m}$. The decomposition in modes of the truncated series permits identification of displacements which are dangerous for the multipolar content and how they could be cured. An application to compare two designs of the LHC dipole is given.
\end{abstract}

\section{THE LHC DIPOLE MODEL}

In this paper we deal with the field quality evaluation in the LHC main superconducting dipoles [1]. The magnet cross section should optimize field quality to obtain a sufficiently constant magnetic field to provide a wide dynamic aperture for the beam [2]. Indeed, the nominal design may be difficult to reach under realistic conditions, and coils can be deformed by either manufacturing forces or tolerances in the mechanical parts (see for instance [3]). In this paper we analyse the effect of deformations due to manufacturing and we develop a formalism to work out the dependence of the multipolar coefficients of the field expansion on the deformations.

Mechanical models have been already used to evaluate the displacement of the LHC dipole coils induced by manufacturing and thermal effects. The present model considers one quarter of the two-in-one dipole, i.e. half aperture, with an up-down symmetry that allows only normal multipoles, and a left-right symmetry that imposes the same multipoles on both apertures. Computations are carried out at $1.9 \mathrm{~K}$, and at the injection energy where the problem of the field quality is more relevant. The coil prestress in the midplane, at $1.9 \mathrm{~K}$ is fixed at $40 \mathrm{MPa}$.

The finite element code ANSYS [4] provides the field of deformations induced in the superconducting coil. A Fortran code (ANSIA [5]) produces displaced coordinates for each conductor in a format compatible with ROXIE [6]. The latter evaluates the magnetic field as a multipolar expansion. The model used to carry out the magnetic computations only considers the effect of deformations on the nominal geometry; the contribution due to iron deformations can be assumed to be negligible.

The mechanical model includes the nonlinear elasticity of the coil [7, 8] as obtained from experimental data [9].

\footnotetext{
*Email: walter.scandale@ cern.ch
}

Particular care was taken in preparing the representation of the elastic modulus of the copper wedges and insulation, that are considered a unique material in the model. Our computations show that the multipoles exhibit a strong dependence on mechanical deformations. Experiments to verify our results are in preparation.

\section{TOOLS FOR THE DEPENDENCE OF MULTIPOLES ON DEFORMATIONS}

The deformation is a field of $2 \mathrm{D}$ vectors that is evaluated through the finite element code ANSYS on a finite set of nodes in a 2D space (the magnet cross section). Since this vector field is rather smooth, we propose to parametrize the deformations by interpolating them with low-order Taylor and Fourier series. In this way all the information relative to the deformations can be reduced to a few coefficients, thus simplifying the analysis of the relation between deformations and multipoles. We numerically estimate the influence of single deformation modes on the multipoles, thus identifying the most dangerous.

Since deformations are implemented in ROXIE as shifts in the position of the inner part and of the outer part of the conductor, we consider a field of deformation defined over four radii $\left(r_{1}, r_{2}, r_{3}, r_{4}\right)=(28,43.4,43.9,59.3) \mathrm{mm}$ that surround the inner and the outer layers of the coils; the field then depends on the discrete variable $r_{i}$ and on the continuous angular variable $\phi$. Deformations are then decomposed into a radial $d r\left(r_{i}, \phi\right)$ and a tangential $d p\left(r_{i}, \phi\right)$ component.

Radial deformations are well interpolated by the sum of the first orders of an even Fourier series in the azimuthal variable $\phi$

$$
d r\left(r_{i}, \phi\right)=\sum_{k=0}^{K} a_{k, i} \cos (k \phi) \quad i=1,4 .
$$

Here the index $i$ runs over the four concentric circles that surround inner and outer coils, and $k$ runs over the modes. The mode zero $a_{0, i}$ gives rise to a contraction or to a dilatation of the coil; the mode one $a_{1, i}$ produces an horizontal shift in the coil, whilst higer order modes correspond to radial waves of deformations with decreasing period. The up-down symmetry is responsible of the absence of sine terms in the Fourier series.

Tangential deformations are well interpolated by low order odd Taylor series in each half of the aperture

$$
d \phi\left(r_{i}, \phi\right)=\sum_{j=0}^{J} b_{j, i} \phi^{2 j+1} \quad i=1,4 \quad \phi \in[-\pi / 2, \pi / 2]
$$




$$
d \phi\left(r_{i}, \phi\right)=\sum_{j=0}^{J} c_{j, i} \phi^{2 j+1} \quad i=1,4 \quad \phi \in[\pi / 2,3 \pi / 2]
$$

The mode zero $b_{0, i}$ and $c_{0, i}$ corresponds to tangential compression or dilatation, uniform along the azimuth. Higher order modes correspond to tangential waves of compressions and dilatations. Also in this case, the up-down symmetry implies the presence of odd terms only.
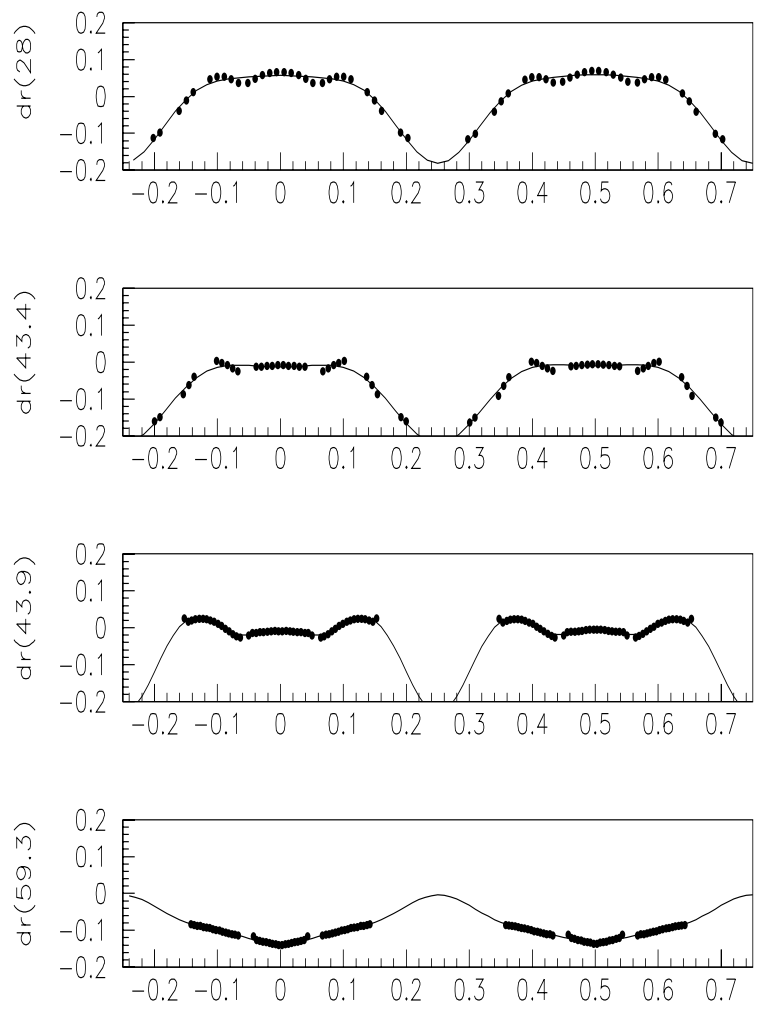

$\varphi / 2 \pi$

Figure 1: Plot of radial deformations for each of the four coil contours, stainless steel collars. Dots: ANSYS values. Lines: interpolation.

In Figs. 1 and 2 we show the radial and tangential deformations evaluated through ANSYS (dots) and the interpolation (solid line) with seven radial modes and two tangential modes ( $K=6$ and $J=1)$. If the displacements evaluated through ANSYS are replaced by the interpolating field, the resulting multipoles agree with the ANSYS results (see Table 1, last two rows). This means that low order Taylor and Fourier series can take into account the part of the deformations that are relevant to evaluate the multipoles.

The contribution of each mode to the multipoles is given in Table 1. For symmetry reasons, even radial modes only contribute to odd multipoles, and odd radial modes to even multipoles. One can distinguish two main effects.

- Radial deformation of order 2 (i.e., circles are de-
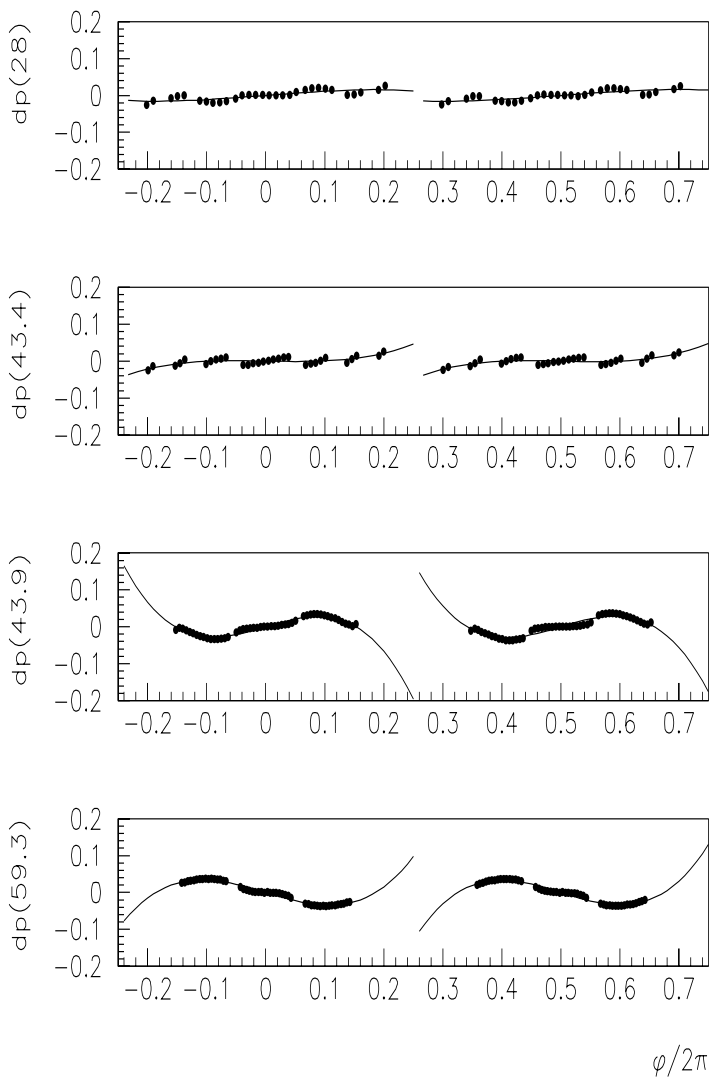

Figure 2: Plot of tangent deformations for each of the four coil contours, stainless steel collars. Dots: ANSYS values. Lines: interpolation.

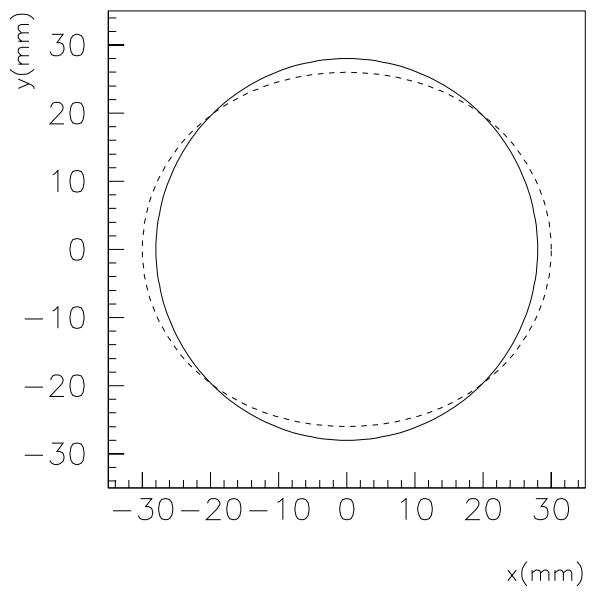

Figure 3: Plot of radial deformations, mode 2, in cartesian coordinates. Undeformed circle (solid line) versus deformed circle (dotted line). 
Table 1: Contribution of radial and tangential modes to the multipoles shift induced by deformation, total effect of 7 radial and 2 tangential modes, and comparison with ANSYS results. Multipole shifts are given in units of $10^{-4}$ at $R_{\text {ref }}=17 \mathrm{~mm}$.

\begin{tabular}{|l|c|c|c|c|}
\hline & $b_{2}$ & $b_{3}$ & $b_{5}$ & $b_{7}$ \\
\hline radial & & & & \\
$\mathrm{k}=0$ & 0.00 & 0.11 & -0.06 & 0.01 \\
$\mathrm{k}=1$ & 0.05 & 0.00 & 0.00 & 0.00 \\
$\mathrm{k}=2$ & 0.00 & -2.98 & 0.18 & -0.04 \\
$\mathrm{k}=3$ & 0.08 & 0.00 & 0.00 & 0.00 \\
$\mathrm{k}=4$ & 0.00 & 0.53 & 0.69 & 0.01 \\
$\mathrm{k}=5$ & 0.00 & 0.00 & 0.00 & 0.00 \\
$\mathrm{k}=6$ & 0.00 & 0.02 & -0.05 & -0.07 \\
tang. & & & & \\
$\mathrm{j}=0$ & 0.04 & 0.17 & -0.05 & 0.02 \\
$\mathrm{j}=1$ & -0.01 & 0.03 & -0.01 & 0.00 \\
& & & & \\
total & 0.16 & -2.12 & 0.70 & -0.07 \\
\hline ANSYS & 0.16 & -2.19 & 0.59 & -0.08 \\
\hline
\end{tabular}

formed into ellipses, see fig. 3) have a strong effect on the sextupole $(\approx-3$ units) and on the decapole $(\approx 0.2$ units $)$.

- Radial deformation of order 4 gives rise to 0.5 units of $b_{3}$ and 0.7 units of $b_{5}$.

Simulations have also shown that the radial deformations are rather well decoupled with the tangential one. In Table 1 the effect of the tangential modes seems to be very limited (only some contribution to $b_{3}$ ): in our computations we fixed the coil length to an optimal value that allows to recover the nominal length under deformations, at $1.9 \mathrm{~K}$. Indeed, a non optimized coil length produces tangential deformations that strongly affects the field multipoles.

- Tangent deformations due to a coil length different from the nominal one have a large influence on $b_{3}, b_{5}$ and $b_{7}$. Simulations show that a $0.1 \mathrm{~mm}$ longer coil gives rise to around -3 units of sextupole, and 0.5 of decapole.

Therefore, the determination of the exact coil length under manufacturing forces and at cold is crucial to obtain a correct estimate of the absolute value of the multipoles. Other contributions of the deformations are not very relevant, giving rise to multipole shifts of less than 0.1 units. In particular, the following effects are negligible:

- The effect of the first order (see Table 1, mode $j=1$ ) tangential mode (tangetial waves fo compression and dilatation) is negligible.

- The radial mode of order zero (see Table 1, mode $k=0$ ), i.e. the radial shrinkage due to low temperature, is negligible.
- Odd radial modes (see Table 1 , mode $k=1,3,5$ ), i.e. modes related to left-right asymmetries, are very low. Therefore, deformations have negligible effect on even multipoles.

\section{CONCLUSIONS}

We have analysed the variation of the field quality in the superconducting LHC dipoles due to deformations of the coils with respect to the nominal design.

We propose a method, based on interpolation, to reduce the deformation field to a few coefficients of radial and tangential modes. This leads to a reduction in the complexity of the problem and to relate modes to multipoles. We are able to identify which modes are more dangerous and which are negligible. We showed that the effect of deformation on multipoles is relevant, and that the main contributions come from radial deformations of order two and four (see for instance fig. 3). Moreover, a systematic difference in the coil length between the nominal and the real one (i.e., at $1.8 \mathrm{~K}$ and under deformations) leads to additional multipole shifts. These effects are uncoupled.

We wish to acknowledge A. Desirelli, P. Fessia, D. Leroy, M. Modena, D. Perini, Prof. Rebora, G. Spigo and R. Vuillermet for their help in the definition and in the understanding of the mechanical model. We also thank S. Russenschuck for providing the ROXIE code and the files of the magnetic model.

\section{REFERENCES}

[1] The LHC study group, CERN 95-05 (1995).

[2] C. Wyss et al., CERN LHC-MMS 98-198 IT 2325 (1998).

[3] R. Bartolini, P. Ferracin, P. Fessia, W. Scandale, E. Todesco, ', in Sixth European Accelerator Conference, edited by C. Petit-Jean Genaz (IoP, New York, 1998) pp. in press.

[4] ANSYS 5.4, licensed by and trademark of Swanson Analysis Inc., Houston, PA, USA.

[5] E. Todesco, private communication.

[6] S. Russenschuck, LHC Project Report 159 (1997).

[7] W. Scandale, E. Todesco, P. Tropea, in preparation.

[8] P. Tropea, A. Rebora, E. Todesco, CERN LHC-MMS Internal Note 99-04 (1999).

[9] D. Tommasini, S. Russenschuck, L. Bottura, in: Proceedings of the first international conference of Roxie users, CERN Yellow Report, in press. 